\title{
Dynamical Calculations of Electron Diffraction for Crystals with incommensurate modulation
}

Lijun Wu and Yimei Zhu

Condensed Matter Physics and Materials Science Department, Brookhaven National Laboratory, Upton, New York, 11973, USA

Quantitative electron diffraction (ED), e.g. convergent beam electron diffraction, has been used to determine atomic structure and charge distribution for crystals. Due to strong dynamic effect of the electron beam, it is necessary to calculate the ED with multiple scattering for quantitative analysis. However, so far the ED calculations are limited to crystals without modulation or with commensurate modulation. Here, we present the ED calculations for crystals with incommensurate modulation based on Bloch wave method that has been widely used to calculate ED for simple crystals. 